\title{
Effect of Work Stress on EEG Activity in Medical Professionals
}

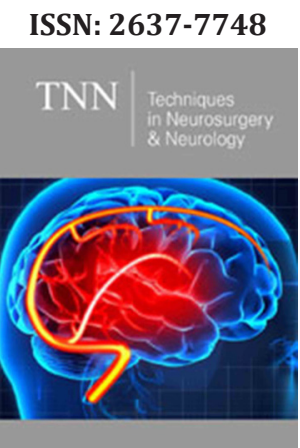

*Corresponding author: Adel A H Mahmoud, 1Pediatric Neurology Department, Neurosciences Center, King Fahad Medical City, Riyadh, Saudi Arabia

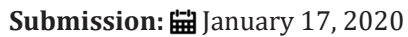

Published: 鈿January 24, 2020

Volume 3 - Issue 2

How to cite this article: Adel A H M, Sajjad A, Lamya J, Sumayyia M, Murtada E M. Effect of work stress on EEG activity in medical professionals. Tech Neurosurg Neurol.3(2). TNN.000558.2020. DOI: 10.31031/TNN.2020.03.000558

Copyright@ Adel A H Mahmoud, This article is distributed under the terms of the Creative Commons Attribution 4.0 International License, which permits unrestricted use and redistribution provided that the original author and source are credited.

\section{Adel A H Mahmoud*1, Sajjad Ali², Lamya Jad ${ }^{1}$, Sumayyia Marar ${ }^{3}$ and Murtada El Mubarak ${ }^{2}$}

${ }^{1}$ Pediatric Neurology Department, Neurosciences Center, King Fahad Medical City, Riyadh, Saudi Arabia

${ }^{2}$ Neurophysiology Department, Neurosciences Center, King Fahad Medical City, Riyadh, Saudi Arabia

${ }^{3}$ Research Center, King Fahad Medical City, Riyadh, Saudi Arabia

\begin{abstract}
Purpose: This study aimed to examine potential differences in electroencephalogram (EEG) activity according to workload stress suffered by medical professionals in our institute, King Fahad Medical City (KFMC) in Riyadh, Kingdom of Saudi Arabia (KSA) during working days relative to non-working days.
\end{abstract}

Methods: A prospective cohort study of 73 medical professionals from KFMC was conducted. EEG recordings were gathered twice per participant-once during working (stress) day and again during the non-working (rest) day.

Results: The p-value was calculated as 0.988 . A positive relationship between EEG activity during days of work and that found during non-working days could not be established in our cohort study.

Conclusion: In our study, no evidence of a positive relationship was found between EEG findings in medical professionals on high-stress working days compared to low-stress non-working days. This does not exclude the possibility of presence of a true relation, as the period between the EEGs done on duty days and off days was short and not allowing for relief and relaxation effects to occur.

Keywords: Potential differences; Electroencephalogram; Relaxation effects; Psychological stress; Behavior; Cognitive processes; Systemic diseases; Psychological; Metabolic diseases; Institutional Review Board; Polyrhythmic

\section{Introduction}

Many people experience stress daily. While there is a close relationship between stress and mental health, psychological stress and its associated emotions, such as anger, anxiety, and depression, can also exert harmful effects on physical health. An electroencephalogram (EEG) is an electrophysiological monitoring device for recording electrical activity within the cortex. The EEG was originally developed to evaluate the human brain cortical activity. The nature of background activity in human brains, and how it reflects behavior and cognitive processes, has been of foremost interest to scientists since Hans' discovery of the alpha rhythm [1]. Recently, certain oils were found to decrease blood pressure, heart rate, and skin temperature, with accompanying autonomic arousal and subjective reports of feeling refreshed [2]. These oils may simultaneously increase the power of theta $(4-8 \mathrm{~Hz})$ and alpha $(8-13 \mathrm{~Hz})$ rhythms. Progressive muscle relaxation was found to be associated with an increase of posterior theta $(3.5-7.5 \mathrm{~Hz})$ and a decrease of mid-frontal beta- 2 band $(20-29.5 \mathrm{~Hz})$ [3]. Taking these facts into consideration, we aimed to examine possible changes in EEG activity in response to varying degrees of work load (i.e., stress) in our facility's medical professionals.

\section{Methods}

A prospective cohort study of 73 medical professionals from KFMC was performed, with sample size having been determined by a biostatistician as sufficient to determine the relationship between EEG activity of participants during working days relative to nonworking days. A significant proportion of participants comprised volunteer nurses. The 
inclusion criteria consisted of medical professionals employed at KFMC lacking any of the following exclusion criteria: medical professionals with neurological or systemic diseases that may affect EEGs (e.g., endocrinological, psychological, or metabolic diseases) or consumption of medication that might affect EEG findings. Informed consent was obtained. Participants' identities remained anonymous and their EEG results were strictly confidential. The study was conducted over 18 months between 2016 and 2018, during which period two EEG studies were performed for each participant, one at the end of a standard working day and the other during a weekend or another non-working day. The EEGs were reported by our EEG reading consultants who were blinded to experimental conditions. Three technicians contributed to recording and blinding the EEGs. After the completion of 146 EEGs, the findings were analyzed, and comments were documented. The 2 reports per candidate were objectively compared, the comparison was tabulated, and the statistical analyses were performed by a biostatistician. Only one volunteer was exempt from inclusion in the study, and no participant requested voluntary withdrawal.

\section{Ethical Considerations}

The study was conducted according to international standards of Good Clinical Practice (International Conference on Harmonization guidelines), applicable government regulations, and institutional research policies and procedures. Our local Institutional Review Board (IRB) approved the protocol and its amendments. All subjects signed a consent form describing the study. This consent form provided sufficient information for subjects to make an informed decision about their participation.

\section{Data Collection}

Every 2 EEGs performed for a given test subject were read by the same electroencephalographer. The main parameters are described below:

\section{Background activity}

Both the background activity and the changes that appear in the features of the tracing are described in terms of frequency, amplitude, wave shape (morphology), symmetry synchrony, location, continuity, and reactivity.

\section{Frequency}

Frequency bands were used to describe the activity irrespective of the activity's location.

\section{Rhythmicity, periodicity, and synchrony}

The term monorhythmic was used when a activity showed oscillations of a single frequency. When multiple frequencies were present concurrently, the term polyrhythmic was used. The term periodic applies to EEG waves or complexes recurring at approximately regular intervals; usually, the intervals vary from one to several seconds.

\section{Amplitude}

The voltage was expressed in microvolts based on a peak-topeak measurement while defining the sensitivity. The amplitude was quantified rather than relying solely on qualitative terminology (e.g., low, medium, and high amplitude) to increase objectivity. The term "suppression" was used when little or no electrocerebral activity could be discerned in each tracing. The montage was reported. Paroxysmal activity in the recording denotes activity of much different synchrony, rhythmicity, and amplitude than dominant activity that occurs acutely and transiently. Symmetry refers to the simultaneous appearance of morphologically identical waveforms in areas on the ipsilateral or contralateral sides of the head. Activity was described as continuous or intermittent depending on the percentage of time it is present. It was deemed continuous when it occurred without interruption for prolonged periods of time, and discontinuous or intermittent when it appeared only occasionally.

\section{Artifacts}

The study examined environmental, instrumental, and electrode artifacts. Furthermore, physiological artifacts, including the heart, the muscles of the head and neck, the eyes, and the skin, and electrocardiogram (ECG) artifacts, EMG artifacts, and eyemovement artifacts were also examined. The data were collected and entered Excel spreadsheets. We used the Chi-square statistical method to obtain the $p$ value, since this study utilizes binary values (True $=1$, False $=0$ ). The $\mathrm{p}$ value indicated that a conclusive correlation could not be established between EEG findings and workload presence, with working days being associated with stress and non-working days being associated with the absence of stress.

\section{Results}

An expert statistician performed all statistical analyses, and comparative elements were assigned numbers to render the evaluation more thematic. Females represented $71.43 \%$ of the total sample while males accounted for $28.57 \%$. The mean age of the sample was $37.72 \pm 8.06$ years. The change between EEGs done on days of work compared to days of rest was more pronounced in females relative to males [4-6]. The amplitude change in background was more pronounced in females. No correlation was found for handedness effects ( $\mathrm{p}=0.988$ ) (Figures 1-3).

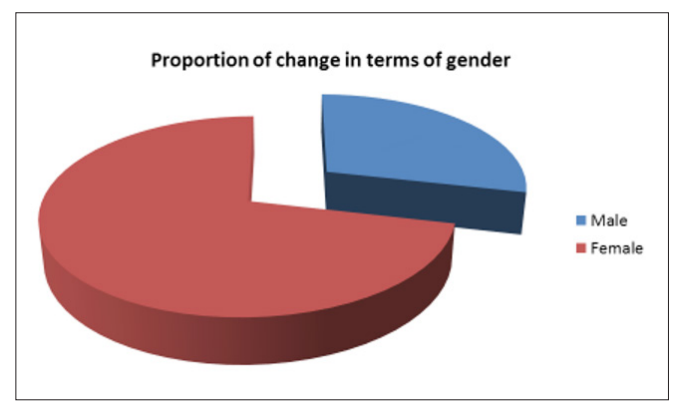

Figure 1: Proportion of change in terms of gender. 


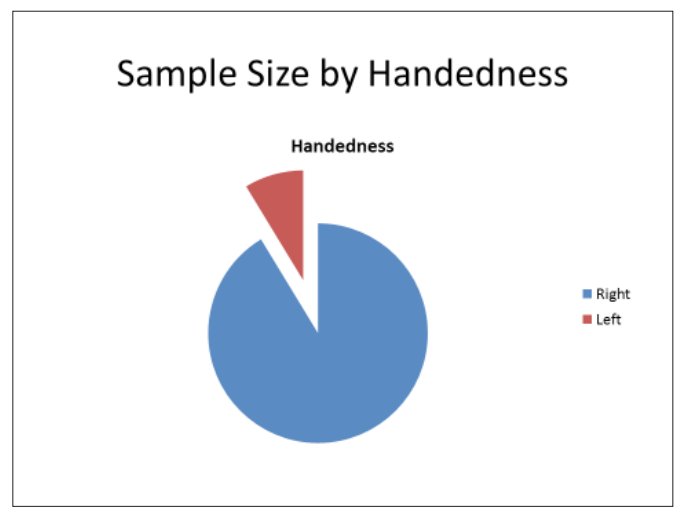

Figure 2: Handedness sample size.

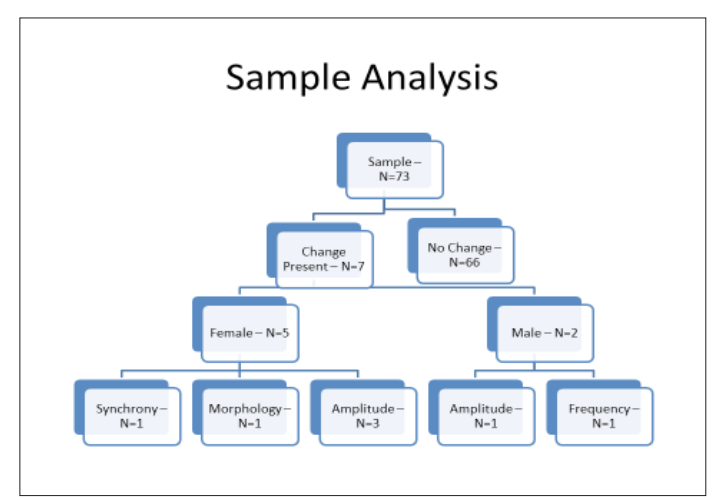

Figure 3: Analysis of samples.

\section{Discussion}

Stress is a major contributing factor to the etiology and manifestations of chronic disorders. It may affect one's motivation to work, performance at work, and general attitude towards life. Thus, stress can strain social and medical resources, increase economic losses and decrease productivity and staff performance. Therefore, instantiation of precautionary measures to reduce stress and adequate management of its precipitating circumstances are essential for both individual and public health. EEG is an electrophysiological monitoring method, first devised by Richard Caton (1842-1926) in 1875, to record the electrical activity of the brain. He presented his findings of electrical phenomena of the exposed cerebral hemispheres of rabbits and monkeys in the British Medical Journal [7]. EEG is typically noninvasive; the electrodes are placed on the scalp, enabling the recording of voltage fluctuations resulting from ionic current within neurons [6]. Lavender oil has been found to significantly decrease blood pressure, heart rate, and skin temperature, also giving rise to autonomic arousal and feelings of reinvigoration [2]. It also increases the power of theta $(4-8 \mathrm{~Hz})$ and alpha $(8-13 \mathrm{~Hz})$ brainwaves, with more scattering power in the alpha range in temporal and central areas. Additionally, monochord sounds and progressive muscle relaxation reduce anxiety and improve relaxation; during chemotherapy, these were associated with an increase in posterior theta $(3.5-7.5 \mathrm{~Hz})$ rhythm and a decrease of the mid-frontal beta- 2 band $(20-29.5 \mathrm{~Hz})$ [3].
Many people experience stressors during their everyday life, and evidence has accumulated that emotions related to psychological stress, such as anger, anxiety, and depression, can have a profound impact on physical health. Indeed, chronic psychological stress can change the responsiveness of central-peripheral regulatory systems $[7,8]$ potentially rendering them less efficient or adaptive for supportive health functions. Stress also affects one's desire to work, performance at work, and broader attitude towards life itself [9]. Furthermore, stress is implicated in $75 \%$ of all heart and brain blood vessel diseases [10]. It is apparent, therefore, that stress can increase and expedite social and economic losses and thereby decrease a country's competitiveness [11]. The need for qualifying and quantifying stress, where possible, is warranted to implement effective measures that can aid workers mitigate or eliminate stressors, leading to expected positive outcomes in work and social life. There are many bio-signal channels by which stress can be potentially quantified, including ECG, EEG, and skin conductance response. ${ }^{11}$ Determining the stress level of any individual, however, can be difficult. In healthy people not experiencing adverse stress, a balance and flexibility of response is seen between the sympathetic and parasympathetic arms of the autonomic nervous system. Studies on brain activity patterns under stressful conditions have largely focused on stress generated by words, examinations, noise, and mental tasks $[12,13]$. EEG is a good technique for determining the relationship of stress concerning suppression of alpha waves and a promotion of theta waves [14]. Alpha waves are more active in occipital and frontal regions of the brain, and such waves are associated with passivity of the brain; when the subject is stressfree and the brain is not engaged in an activity, alpha waves are dominant. However, under stressful conditions, the intensity of alpha waves drops, indicating the change in response to stress. Beta waves show varying behaviors under different frequencies in different parts of the brain, and theta wave intensity increases under stress or psychological pressure [15-17].

A study was conducted to examine the correlation between heart rate variability (HRV) and elevated beta activity at anterior temporal sites, as well as the correlation between salivary cortisol and beta activity. ${ }^{11}$ The results demonstrated that participants with relatively low HRV had relatively high levels of beta activity in premotor regions of the cortex. Participants with a higher level of salivary cortisol also had a higher level of beta activity. The authors suggested the presence of close relationships between EEG, ECG, and salivary cortisol indicative of chronic stress. Salivary cortisol was associated with relatively high levels of beta power at anterior temporal sites. A study conducted at University of Cape Town, South Africa, has elucidated the effect of HRV biofeedback on measures of EEG during, and immediately after, biofeedback. The results suggested that a single session of HRV biofeedback after a single training session was associated with changes in EEG suggestive of increased internal attention and relaxation both during and after the intervention. However, the comparative intervention was associated with changes suggestive of increased mental effort and possible anxiety during and after the intervention [11]. Another 
study used encephalography to examine the effect of hyperthermia on brain electrical activity during prolonged exhaustive exercise in a group of sedentary women. The study concluded that exhausting work in high temperatures induced a change in gross brain activity (alpha/beta ratio) compared to a longer, less thermally demanding exposure [11]. Darwaj et al. [5] studied the effect of stress on sleep to find that duration of rapid eye movement (REM) sleep is reduced during stress while its intensity increases significantly [5]. Failure of our study to obtain a statistically significant correlation between work stress and EEG activity changes may not reflect reality. The rest period, for instance, may have been insufficient in duration to study the effect of rest on EEG activity. Also, the finding that females evinced a more pronounced effect with more changes in EEG activity during rest days compared to working days might constitute a reflection of female sensitivity to stress or a more favorable reaction to rest compared to males. Furthermore, although the sample size was reasonable as per statistical calculations, it would have benefitted from increased numbers of recruited candidates. Despite these findings, we still suspect that stress influences cortical activity, which may require greater numbers and longer periods between stress and rest to bring the phenomenon to light.

\section{References}

1. Haas LF (2003) Hans Berger (1873-1941), Richard Caton (1842-1926), and electroencephalography. J Neurol Neurosurg Psychiatry 74(1): 9.

2. Sayorwan W, Siripornpanich V, Piriyapunyaporn T, Hongratanaworakit T, Kotchabhakdi N, et al. (2012) The effects of lavender oil inhalation on emotional states, autonomic nervous system, and brain electrical activity. J Med Assoc Thai 95(4): 598-606.

3. Lee EJ, Bhattacharya J, Sohn C, Verres R (2012) Monochord sounds and progressive muscle relaxation reduce anxiety and improve relaxation during chemotherapy: A pilot EEG study. Complement Ther Med 20(6): 409-416.

4. Tanzer IO (2006) Numerical modeling in electro- and magnetoencephalography. TKK Dissertations 26: 192.
5. Darwaj B, Smyk K, Czochra T (1977) Effect of stress on sleep characteristics in humans. Neurol Neurochir Pol 11(2): 145-151.

6. Da Silva FL Basic Principles, clinical applications, and related fields.

7. Fuchs E, Flügge G (1995) Modulation of binding sites for corticotropinreleasing hormone by chronic psychosocial stress. Psychoneuroendocrinology 20(1): 33-51.

8. Fuchs E, Uno H, Flügge G (1995) Chronic psychosocial stress induces morphological alterations in hippocampal pyramidal neurons of the tree shrew. Brain Res 673(2): 275-282.

9. Sauter SL, Hurrel JJ Jr, Fox HR, Tetrick LE, Barling J (1999) Occupational health psychology: An emerging discipline. Ind Health 37(2): 199-211.

10. Belkic KL, Landsbergis PA, Schnall PL, Baker D (2004) Is job strain a major source of cardiovascular disease risk? Scand J Work Environ Health 30(2): 85-128.

11. Daly M, Delaney L, Doran PP, Harmon C, MacLachlan M (2010) Naturalistic monitoring of the affect-heart rate relationship: A day reconstruction study. Health Psychol 29(2): 186-195.

12. Matsunami K, Homma S, Han XY, Jiang YF (2001) Generator sources of EEG large waves elicited by mental stress of memory recall or mental calculation. Jpn J Physiol 51(5): 621-624.

13. Seo DC, Li K (2009) Effects of college climate on students' binge drinking: Hierarchical generalized linear model. Ann Behav Med 38(3): 262-268.

14. Natasha P, Nikitha T, Bhatter S, Subhashish K, Harshitha R (2018) Detection of mental stress using EEG signals. IJET 4(1).

15. Ryu K, Myung R (2005) Evaluation of mental workload with a combined measure based on physiological indices during a dual task of tracking and mental arithmetic. Int J Ind Ergonom 35(11): 991-1009.

16. Harmony T, Fernández T, Silva J, Bernal J, Díaz Comas L, et al. (1996) EEG delta activity: An indicator of attention to internal processing during performanceof mental tasks. Int J Psychophysiol 24(1-2): 161171.

17. Tong S, Thakor NV (2009) Quantitative EEG analysis methods and clinical applications. Artech House pp. 300. 